\title{
Determinants of early marriage among female children in Amhara Region, Ethiopia
}

\author{
Abay Kassa Tekile, Ashenafi Abate Woya, Garoma Wakjira Basha
}

Department of Statistics, Science College, Bahir Dar University, P.O. Box: 79, Bahir Dar, Ethiopia.

\author{
Emails: \\ ashu.abate@gmail.com(Ashenafi Abate Woya); garewa2010@gmail.com(Garoma Wakjira Basha)
}

\begin{abstract} to practice early marriage compared to parents who knew the legal marital age. cant determinants of early marriage practice.

Keywords: Early Marriage, Female, Amhara Region, Ethiopia.

DOI: https://dx.doi.org/10.4314/ahs.v20i3.22

\section{Introduction}

Child early marriage is defined as "any marriage carried out below the age of 18 years, before the girl is physically, physiologically, and psychologically ready to shoulder the responsibilities of marriage and childbearing. It therefore has major consequences for public health, national security, social development, human rights, economic development and gender equality ${ }^{1}$. Similarly, the age at first marriage is defined as the age at which the respondents began living with her/his first partner².
\end{abstract}

Background: Early Marriage is one of the global problems that undermine the personal development and the rights of women seriously. It is delicate among the developing countries such as Ethiopia. It has major consequences for public health, national security, social development, human rights, economic development, and gender equality.

Methods: The analyzed data were obtained from the 2016 EDHS and 1120 samples were considered in this analysis. Both bivariate and multivariable binary logistic regression model were used to identify the determinants of early marriage practice. Results: The prevalence of early marriage practice was $48.57 \%$ in the study area. The odds of early marriage practice were 2.04(AOR $=2.04,95 \% \mathrm{CI}: 1.88,2.45)$ times higher among rural residents compared to urban. The odds of early marriage practice was $0.94(\mathrm{AOR}=0.94,95 \% \mathrm{CI}: 0.57,1.98)$ times lower among women who had primary education compared to uneducated women. Those who did not know the legal marital age were $1.61(\mathrm{AOR}=1.61,95 \% \mathrm{CI}: 1.26,2.07)$ times more likely

Conclusion: Education level, family monthly income, residence, literacy level and knowledge of legal marital were signifi-

Cite as: Tekile $A K$, Woya $A$ A, Basha GW. Determinants of early marriage among female children in Ambara Region, Ethiopia. Afri Health Sci. 2020;20(3): 1190-1195. bttps://dx.doi.org/10.4314/abs.v20i3.22

The extent of early marriage varies between countries and regions. The highest rates are reported in South Asia and sub-Saharan Africa, where $44 \%$ and $39 \%$ of girls, respectively, were married before the age of 18 years. Data from 33 countries showed that trends in marriage

\section{Corresponding author:}

Abay Kassa Tekile,

Department of Statistics,

Science College, Bahir Dar University,

P.O. Box: 79, Bahir Dar, Ethiopia.

Email: abaystat@gmail.com indicate limited change since the International Conference on Population and Development ${ }^{3}$. More over 27 per cent were in East Africa and 20 percent in Northern and Southern Africa ${ }^{4}$. As the current estimates of the Convention on the Elimination of all forms of discrimination against women showed, approximately 82 million girls in the world between 10-17 years will be married before they reach 18 years; and of the 331 million girls aged 10-19 in developing countries,163 million will be married before they are 20 years ${ }^{5}$.

A study in Gojjam and South Wollo zones of Amhara region indicated that early marriage is highly prevalent. The prevalence is higher for women than men. About $49 \%$ of women were married before age 15 and about $83 \%$ were married before age 18 years $^{6}$. By 2015, the prevalence of female early marriage was $76.7 \%$ in Amhara region, North Ethiopia. Females who did not know the legal marital age were 12 times more likely to practice early marriage compared to those who knew the legal marital age?

A study by marital relations and Intimate Partner Violence in Ethiopia showed $70 \%$ of respondents had married before age 15 years and 30\% had married at 
ages 15-17 years. Among those who married before 15 years of age, $82.2 \%$ were from rural residences. This study also showed that rural residence added more risk for early marriage. Rural residence was associated with nearly a threefold elevation in the odds of marriage at ages $15-17$ years $^{8}$.

A study by Pathfinder International in Amhara region showed that the prevalence of girls' early marriage was $81.8 \%$. Moreover, about $44 \%$ of urban and $53 \%$ of rural ever-married women were married between 12-15 years of age. The proportion marrying between age 16 and 17 years was $14.5 \%$ in urban and $15.5 \%$ in rural areas?

In Ethiopia, female child early marriage is seen as a way to improve the economic status of the family, to strengthen ties between families, to ensure that girls are virgins when they marry, and to avoid the possibility of a girl reaching an age where she is no longer desirable as a wife. The practice of female child early marriage is now understood to have very harmful effects on the health, psychological, physiological and socio-economic well-being of young girls (as well as for the newborns). None the less, this knowledge is not broadly shared across most of the population ${ }^{10}$.

In Amhara region of Ethiopia, about $80 \%$ of girls are married before they are 18 years old, and the most common age for a girl to marry is 12 years old. Child marriage is rooted in religious and cultural tra $\neg$ ditions based on protecting a girl's honor since sex before marriage is seen as an extremely shameful act. A girl's worth is therefore based on her virginity and her role of being a wife and mother ${ }^{11}$.

All relevant laws of Ethiopia established a legal minimum age at marriage of 18 years for boys and girls. In fact, much of the education on early marriage prevention clearly indicates that the legal minimum age for marriage is 18 years for both girls and boys. However, a study in Amhara region shows clearly that the general public's definition of early marriage for girls uses a much lower cut-off than the legal definition. This indicates that the cut-off age for defining early marriage for female adolescents was often ignored with many marriages occurring before age 15.3 years ${ }^{12}$.

The prevalence of early marriage in the Amhara region is still high. The findings of this study will make an input for the policy makers and planners in the area as well as the regional government to respond to early marriage at all levels of governmental and non-governmental sectors. Furthermore, it will help as an initiative for further investigation and intervention in the area regarding early marriage for those who will be interested in studying its consequences and related issues. Therefore, the goal of this study was to determine the prevalence and factors associated with early marriage in the Amhara region, North Ethiopia.

\section{Methods}

\section{Study design and sampling}

The dataset used in this study was obtained from the Ethiopia Demographic and Health Survey (EDHS) conducted by Central Statistics Agency (CSA) in 2016. The survey utilized a multistage cluster sample and was designed to obtain and provide information on the basic indicators of the health and demographic variables. The study design was cross-sectional, in which the data on the independent and outcome variables is collected at the same point in time.

\section{Target population}

The target population was all female community members in selected cities of Amhara Region who practiced marriage.

\section{Dependent variable}

Female child Marriage (classified as either below 18 or 18 and above).

\section{Independent variables}

Age, Education, Religion, income, ethnicity, literacy level and residence area(urban/rural).

\section{Data analysis}

The data were extracted, edited, recorded, and analyzed by using SPSS version 23 for Windows. Both bivariate and multivariable binary logistic regressions were done. Bivariate logistic regression was performed and variables with a $\mathrm{p}$-value less than 0.25 were transported into multivariable binary logistic regression analysis to identify the determinants of early marriage among female children. Finally, variables with p-values $<0.05$ in the multivariable logistic regression modewere taken as statistically significant. The association was reported as adjusted odds ratios with $95 \%$ confidence intervals.

\section{Binary logistic regression model}

The binary logistic regression model is used when the dependent variable is a dichotomous and the independent variables are of any kind. This model is mathematically flexible and easily used distribution and it requires fewer assumptions ${ }^{13}$. It is presented as follow:

$\operatorname{logit}\left(\frac{p}{1-p}\right)=\beta_{0}+\beta_{1} X_{1}+\beta_{2} X_{2}+\cdots+\beta_{k} X_{k}$ 
where $\mathrm{p}$ is the probability of being practice early marriage, is a set of independent variables, and $\beta=(\beta 0$, $\beta 1 \ldots \beta \mathrm{k}) \mathrm{T}$ is a vector of unknown coefficients. The quantity to the left of the equal sign is the log of the odds of early marriage in the binary logistic regression. The adequacy of the model was checked by using Hosmer-Lemeshow goodness of fit test.

\section{Results}

A total of 1120 married women were selected for this study. The median age at first marriage for women was 15 years and about $48.6 \%$ of women got married before their 18th birth day in the selected study area. As shown in Table 1, early marriage was significantly associated with women's educational level ( $p$-value $=0.000<0.25$ ). The highest proportion of early marriage was observed in the age group $15-19$ years $(60.6 \%)$. The educational status and early marriage cross tabulation also reveals that the smallest proportion of early marriage $(21.6 \%)$ was observed for those hving higher education while the highest proportion of early marriage was observed for those having no education (51.1\%). The proportion of early marriage was $30.3 \%$ for women that lived in urban and $52.2 \%$ for women who lived in rural. It was also observed that among women that took part in the study, the percentage of early marriage for Orthodox, Protestant, Muslim, and others religion followers were $48.2 \%, 53.8 \%, 50 \%$, and $50.0 \%$, respectively (Table 1 ).

Table 1 Distribution of Early Marriage by Respondents' Characteristics (EDHS, 2016)

\begin{tabular}{|c|c|c|c|c|}
\hline \multirow[t]{2}{*}{ Variables } & \multirow[t]{2}{*}{ Categories } & \multicolumn{2}{|c|}{ Early Marriage } & \multirow[b]{2}{*}{ P-value } \\
\hline & & No $(\%)$ & Yes $(\%)$ & \\
\hline \multirow{7}{*}{$\begin{array}{l}\text { Women's } \\
\text { years }\end{array}$} & $15-19$ & $26(39.4)$ & $40(60.6)$ & \multirow{7}{*}{$0.156 *$} \\
\hline & $20-24$ & $89(57.4)$ & $66(42.6)$ & \\
\hline & $25-29$ & $137(53.7)$ & $118(46.3)$ & \\
\hline & $30-34$ & 102(47.9) & $111(52.1)$ & \\
\hline & $35-39$ & $105(54.1)$ & $89(45.9)$ & \\
\hline & $40-44$ & $60(46.9)$ & $68(53.1)$ & \\
\hline & $45-49$ & $57(52.3)$ & $52(47.7)$ & \\
\hline \multirow[t]{4}{*}{ Education } & Uneducated & $385(48.9)$ & $403(51.1)$ & \multirow[t]{4}{*}{$0.000 *$} \\
\hline & Primary & $120(50.2)$ & $119(49.8)$ & \\
\hline & Secondary & $42(750$ & $14(25)$ & \\
\hline & Higher & $29(78.4)$ & $8(21.6)$ & \\
\hline \multirow[t]{3}{*}{ Literacy level } & Cannot read & $396(50.3)$ & $392(49.7)$ & \\
\hline & Partially & $63(46.7)$ & $72(53.3)$ & \\
\hline & Whole & $116(59.2)$ & $80(40.8)$ & $0.062 *$ \\
\hline \multirow[t]{3}{*}{ Wealth index } & Poor & $203(45.9)$ & $239(54.1)$ & \multirow[t]{3}{*}{$0.001 *$} \\
\hline & Medium & $146(50.0)$ & $146(50.0)$ & \\
\hline & Rich & $227(58.8)$ & $159(41.2)$ & \\
\hline \multirow[t]{2}{*}{ Residence area } & Urban & 129(69.7) & $56(30.3)$ & \multirow{4}{*}{$0.000 *$} \\
\hline & Rural & $447(47.8)$ & $488(52.2)$ & \\
\hline \multirow{4}{*}{ Religion } & Orthodox & $468(51.8)$ & $435(48.2)$ & \\
\hline & Protestant & $6(46.2)$ & $7(53.8)$ & \\
\hline & Muslim & $101(50.0)$ & $101(50.0)$ & \multirow{2}{*}{0.947} \\
\hline & Other & $1(50.0)$ & $1(50.0)$ & \\
\hline \multirow{4}{*}{ Ethnicity } & Amhara & $552(51.4)$ & $521(48.4)$ & \multirow{4}{*}{0.832} \\
\hline & Oromo & $9(45.0)$ & $11(55.0)$ & \\
\hline & Agew-Awi & $7(50)$ & $7(50)$ & \\
\hline & Other & $8(61.5)$ & $5(38.5)$ & \\
\hline
\end{tabular}

\footnotetext{
* P value Significant at $25 \%$
} 
Table 2 shows the results of multivariable logistic regression analysis. The odds of early marriage practice were $0.94(\mathrm{AOR}=0.94,95 \% \mathrm{CI}: 0.57,1.98)$ times lower among women who had primary education compared to uneducated women. Regarding knowledge of legal marital age, those who did not know the legal marital age were $1.61(\mathrm{AOR}=1.61,95 \% \mathrm{CI}: 1.26,2.07)$ times more likely to practice early marriage compared to parents who know the legal marital age.

The literacy level of women was significant for early marriage in the study area. Women who read only parts of the sentence were 0.49 times less likely to get early marriage than who could not read at all. Women who read the whole sentence were $0.36(\mathrm{AOR}=0.36,95 \% \mathrm{CI}$ : $0.24,0.52)$ times less likely to get early marriage than who cannot read at all.

The income level of the family was another significant factor for early marriage in Amhara region. Women who lived in a family with medium monthly income were $0.81(\mathrm{AOR}=0.81,95 \% \mathrm{CI}: 0.60,1.11)$ less likely to get early marriage than who lived in the poor family. Women who lived in a family with rich monthly income were 0.57 less likely to get early marriage than who lived in the poor family. The odds of early marriage practice were $2.04(\mathrm{AOR}=2.04,95 \% \mathrm{CI}: 1.88,2.45)$ times higher among rural residents compared to urban (Table 2).

Table 2 Factors Associated with Early marriage in Amhara region, Ethiopia (EDHS, 2016)

\begin{tabular}{llll}
\hline Variable & Categories & AOR(95\%CI) & P-value \\
& & & \\
\hline Education & Uneducated(ref) & 1 & 0.015 \\
& Primary & $0.96(0.57,1.98)^{*}$ & 0.005 \\
& Secondary & $0.94(0.45,1.94)$ & 0.864 \\
& Higher & $0.69(0.29,1.64)$ & 0.401 \\
Legal age & Yes(ref) & 1 & \\
& No & $1.61(1.26,2.07)^{*}$ & 0.000 \\
Literacy & Cannot read(ref) & 1 & 0.000 \\
& partial & $0.49(0.34,0.72)^{*}$ & 0.000 \\
& Whole sentence & $0.36(0.24,0.52)^{*}$ & 0.000 \\
Wealth & index & 1 & 0.001 \\
level & Medium & $0.81(0.60,1.10)$ & 0.170 \\
Residence area & Rich & $0.57(0.43,0.77)^{*}$ & 0.000 \\
& Urban(ref) & 1 & 0.000 \\
\hline & Rural & $2.04(1.88,2.45)$ & 0.000 \\
\hline
\end{tabular}

*Significant at $5 \%$ significant level, AOR=Adjusted Odds Ratio, CI=Confidence interval

\section{Binary logistic regression}

\section{Assessment of goodness of fit of the model}

The omnibus tests are used to measure how well the model performs. The chi-square tests are used to measure the difference between the initial model and the regression model in terms of a number of correctly classified subjects or it is the change in the -2log-likelihood from the previous step. Since the omnibus test was significant, the model in the final step was consid- ered to be appropriate (Table 3).If the p-value of the Hosmer-Lemeshow Goodness of fit test statistic is greater than $\alpha=0.05$, we fail to reject the null hypothesis that there is no difference between observed and predicted values. The value of Hosmer-Lemeshow statistic had a chi-square value of 2.264 and a p-value of 0.645 indicating that the model had a good fit. This shows that there was no significant difference between the observed and predicted model values and hence the model fits the data well (Table 3). 


\begin{tabular}{|l|l|l|l|l|}
\hline $\begin{array}{l}\text { Omnibus Tests of } \\
\text { Model Coefficients }\end{array}$ & & Chi-square & Df & Sig. \\
\cline { 2 - 5 } & Step & 14.707 & 1 & 0.000 \\
\cline { 2 - 5 } & Block & 55.988 & 5 & 0.000 \\
\cline { 2 - 5 } & Model & 55.988 & 5 & 0.000 \\
\hline \multicolumn{2}{|l}{ Hosmer-Lemeshow Test } & 2.264 & 7 & 0.645 \\
\hline
\end{tabular}

\section{Discussion}

This study examined the prevalence of early marriage and the related factors in the Amhara Region of Ethiopia. The prevalence of early marriage practice was $48.57 \%$ in the study area. This finding was lower than three previous studies in Amhara region, Ethiopia which were $83 \%, 81.85$ and $76.7 \%$ respectively ${ }^{6,7,9}$.

Education level was a significant factor in early marriage. The percentage of early marriage was highest among women with no education. Females with primary education were less likely to get married before reaching the age of age 18 than those with no education. The higher one's educational attainment, the more knowledge females get and understand the best marriage age, and the effect of having an early marriage. This result was supported by the previous study ${ }^{12}$.

The odds of early marriage practice were high for those women who did not know the legal marital age compared to women who know the legal marital age. The odds of early marriage practice were higher among rural residents compared to urban. This finding was similar to previous studies in Amhara region which showed that rural residents were more likely to practice early marriage than urban residents ${ }^{7}$.

The income level was another significant factor for early marriage in Amhara region. Families with medium monthly income were 0.81 times less likely to practice early marriage compared to those having poor monthly income and similarly families with rich income were 0.57 times less likely to get early marriage than poor families. This result was consistent with the previous study which indicates that families with lower monthly income are more likely to practice early marriagthan families with high monthly income ${ }^{7,14}$. This study also revealed that, the odds of early marriage practice were higher among rural residents compared to urban. This finding is in line with a previous study in Ethiopia ${ }^{7}$.

\section{Conclusions and recommendations}

This study established the prevalence and factors as- sociated with early marriage. The authors found that the prevalence of early marriage was still high in the Amhara region, Ethiopia. The study identified education level, family monthly income, literacy, residence area and knowledge of legal marital age as the main factors associated with early marriage practice. Since the education level of women was a significant factor for early marriage, parents and the Ministry of Education should emphasize education of women. In the communities' awareness, regarding the legal marital age, has to be developed.

\section{Authors' contribution}

All the authors participated in proposal development, data extracting, analysis and manuscript writing.

\section{Competing interests}

The authors declare that they have no competing interests.

\section{Acknowledgments}

We would like to acknowledge Central Statistical Agency (Ethiopia) for allowing us to use the Ethiopia Demographic and Health Survey, EDHS 2016 data.

\section{References}

1. Walker JA, Mukisa S, Hashim Y, Ismail H. Mapping Early Marriage in West Africa: a scan of trends, interventions, what works, best practices and the way forward Lagos: Ford Foundation. West Africa Office. 2013.

2. CSA. Ethiopia demographic and health survey, Addis Ababa, Ethiopia and Calverton, Maryland, USA: Central Statistical Agency. 2016.

3. Santhya K.G, Jejeebhoy S.J. Adolescents' health and human rights. Sexual and reproductive health and rights of adolescent girls: evidence from low- and middle-income countries. Global Public Health. 2015;10(2):189221,doi: 10.1080/17441692.2014.986169.

4. Myers J, Harvey R. Breaking Vows: Early and Forced Marriage and Girls' Education. 2011. 
5. Fitch C, Kennedy S, Oakes J, Ruggles S. Marriage Formation and Local Economic Opportunity in the United States: A Comparison of Public-use and Restricted Access Census Data Minnesota Population Center. 2011. 6. Asrese K, Abebe M. Early Marriage in South Wollo and East Gojjam Zones of the Amhara Region, Ethiopia. Humanities and Social Sciences. 2014;2(2):11-6,doi: 10.11648/j.hss.20140202.11.

7. Workineh S, Kibretb GD, Degu G. Determinants of Early Marriage among Female Children in Sinan District, Northwest Ethiopia. Health Science Journal. 2015;9(6):1-7.

8. Erulkar A. Early marriage, marital relations and intimate partner violence in Ethiopia. Int Perspect Sex Reprod Health. 2013;39(1):6-13, doi: 10.1363/3900613.

9. PathfinderInternational. Report on causes and consequences of early marriage in Amhara Region, Addis Ababa, Ethiopia. 2006.
10. CSA. Ethiopia demographic and health survey . Addis Ababa, Ethiopia and Calverton, Maryland, USA: Central Statistical Authority and ORC Macro. 2011.

11. Rodgers B. Child Marriage in Ethiopia and Its Associated Human Rights Violations. Rights Violations Uw Bothell Policy Journal. 2012.

12. Mpilambo J. E, Appunni S.S, Stiegler O.K.N. Determinants of Early Marriage among Young Women in Democratic Republic of Congo. Journal of the Social Sciences. 2017;52(1):82-91, doi: 10.1080/09718923.2017.1322393.

13. Hosmer D, Lemeshow S. Applied Logistic Regression. John Wiley and Sons, Inc., New York. 2000.

14. Rumble L, Peterman A, Irdiana N, Triyana M, EMinnick E. An empirical exploration of female child marriage determinants in Indonesia. BMC Public Health. 2018;18(407), doi: 10.1186/s12889-018-5313-0. 